\title{
Erratum: First axion results from the XENON100 experiment [Phys. Rev. D 90, 062009 (2014)]
}

E. Aprile, F. Agostini, M. Alfonsi, K. Arisaka, F. Arneodo, M. Auger, C. Balan, P. Barrow, L. Baudis, B. Bauermeister, A. Behrens, P. Beltrame, K. Bokeloh, A. Brown, E. Brown, S. Bruenner, G. Bruno, R. Budnik, J. M. R. Cardoso, A. P. Colijn, H. Contreras, J. P. Cussonneau, M. P. Decowski, E. Duchovni, S. Fattori, A. D. Ferella, W. Fulgione, F. Gao, M. Garbini, C. Geis, L. W. Goetzke, C. Grignon, E. Gross, W. Hampel, R. Itay, F. Kaether, G. Kessler, A. Kish, H. Landsman, R. F. Lang, M. Le Calloch, D. Lellouch, C. Levy, S. Lindemann, M. Lindner, J. A. M. Lopes, K. Lung, A. Lyashenko, S. MacMullin, T. Marrodán Undagoitia, J. Masbou, F. V. Massoli, D. Mayani Paras, A. J. Melgarejo Fernandez, Y. Meng, M. Messina, B. Miguez, A. Molinario, M. Murra, J. Naganoma, K. Ni, U. Oberlack, S. E. A. Orrigo, E. Pantic, R. Persiani, F. Piastra, J. Pienaar, G. Plante, N. Priel, S. Reichard, C. Reuter, A. Rizzo, S. Rosendahl, J. M. F. dos Santos, G. Sartorelli, S. Schindler, J. Schreiner, M. Schumann, L. Scotto Lavina, M. Selvi, P. Shagin, H. Simgen, A. Teymourian, D. Thers, A. Tiseni, G. Trinchero, O. Vitells, H. Wang, M. Weber, and C. Weinheimer

(XENON100 Collaboration)

(Received 3 December 2016; published 31 January 2017)

DOI: 10.1103/PhysRevD.95.029904

In our paper, we presented searches for solar axions and galactic axionlike particles (ALPs) in the data collected by the XENON100 experiment (with an exposure of 224.6 days). We recently found a bug in the code to calculate the exclusion limit for galactic ALPs. This resulted in an underestimation of the ALP expected rate, which in turn led to an overly conservative limit, compared to what it should really be.

We corrected the code, and the result of the XENON100 90\% C.L. exclusion limit on galactic ALPs (shown in Fig. 1) was reevaluated. The corrected limit is stronger than the one previously published by approximately a factor of 5 across all masses and sets the best published limit on the axion-electron coupling, $g_{A e}$, in the $(1-40) \mathrm{keV} / \mathrm{c}^{2}$ mass range.

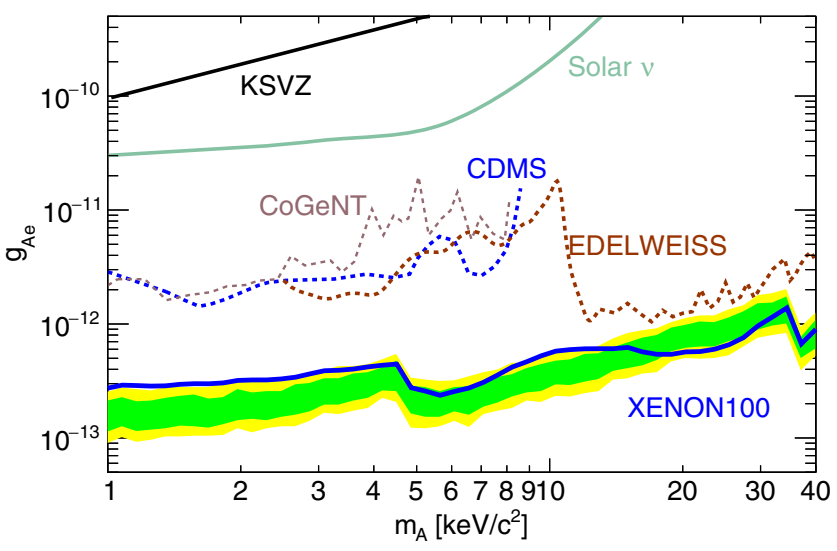

FIG. 1. The XENON100 limit (90\% C.L.) on ALP coupling to electrons as a function of the mass, under the assumption that ALPs constitute all the dark matter in our Galaxy (blue line). The expected sensitivity is shown by the green/yellow bands $(1 \sigma / 2 \sigma)$. The other curves are constraints set by CoGeNT [1] (light brown dashed line), CDMS [2] (blue dashed line), and EDELWEISS-II [3] (brown dashed line). The indirect astrophysical bound from solar neutrinos [4] is represented as a continous light gray line. The benchmark Kim-Shifman-Vainshtein-Zakharov (KSVZ) model is represented by a black line $[5,6]$.

Since the bug affected the ALP expected rate only, the solar axion search result remains unchanged.

[1] C. E. Aalseth et al., Phys. Rev. Lett. 101, 251301 (2008); 102, 109903(E) (2009).

[2] Z. Ahmed et al., Phys. Rev. Lett. 103, 141802 (2009).

[3] E. Armengaud et al., J. Cosmol. Astropart. Phys. 11 (2013) 067.

[4] P. Gondolo and G. Raffelt, Phys. Rev. D 79, 107301 (2009).

[5] J. E. Kim, Phys. Rev. Lett. 43, 103 (1979).

[6] M. A. Shifman, A. I. Vainshtein, and V. I. Zakharov, Nucl. Phys. B166, 493 (1980). 